\title{
LIGHT AND TRANSMISSION ELECTRON MICROSCOPICAL OBSERVATIONS ON RAT SCIATIC NERVE INDUCED BY ELECTROCUTION
}

\author{
DOAA SAFWAT ${ }^{*}$ ADEL SHEHATA ISMAIL ${ }^{* *}$; MAHMUD ABD EL-NASER ${ }^{* *}$; \\ OMAR H. BAYUOMI ${ }^{* * *}$ and SALAH H. AFIFI**** \\ * Department of Forensic medicine and Toxicology, Faculty of Veterinary Medicine, New vally, Assiut University, Assiut, \\ Egypt. \\ ${ }^{* *}$ Department of Forensic medicine and Toxicology, Faculty of Veterinary Medicine, Assiut University, Assiut, Egypt. \\ ${ }^{* * *}$ Electron Microscope unit, Assuit University. \\ ${ }^{* * * *}$ Department of Pathology \& Clinical pathology, Faculty of Veterinary Medicine, Assiut University, Assiut, Egypt
}

Email: Afifi_s_4@hotmail.com

\section{ABSTRACT}

Received at: $27 / 1 / 2014$

The main objective of the present study was to investigate possible alterations of the sciatic nerve of rats exposed to $220 \mathrm{~V}$ for 30 seconds by light and

Accepted: 8/3/2014 transmission electron microscope (TEM). Electric current was applied on the thigh region near the gastrocneamus muscles of rats. The sciatic nerve was taken immediately at 30 and 60 minutes and fixed in $4 \%$ cold glutaraldehyde. They were then processed for both light and TEM. Light microscope showed irregularity of the shape of the nerve bundles and fibers compared to the control. Moreover, annulations of the myelin sheath were detected and mast cell infiltration was observed around the myelin sheath, which suggested a response of the nerve tissue to injury. TEM showed that the myelin sheath of non-exposed rats had no remarkable morphological changes: the thickness was in the range $(1.41 \pm 0.7) \mu \mathrm{m}$. On the contrary, the exposed nerve showed a remarkable increase in thickness $(2.32 \pm 0.8) \mu \mathrm{m}$. The nerves from exposed rats were fragmented either in localized areas of the nerve where they appeared "bulby" or onion-like or in the surroundings of the entire nerve. No changes were observed in Schwann cells. Mast cells were detected around the affected nerve fibers showed empty vesicles, suggested degranulation. These results can be a helpful tool in veterinary forensic medicine.

Key words: Electric current, Forensic toxicology, Microscopy, Morphological changes, Myelin sheath.

\section{INTRODUCTION}

Electroporation damage is the tissue damage induced by the electric field and seems to affect mostly the plasma membrane. It is observed that cell membranes lose their anatomical structure when exposed to trans-membrane potentials above 600800mV (Anders et al., 2001). Chizuka et al. (1983) stated that myelin is a highly specialized multilamellar membrane that results from the elaboration of Schwann cells in the peripheral nervous system. Schwann cell elaborates a highly lipid-rich plasma memberane which wrap tightly around the axon many times it is known that the large, myelinated nerve fibres, should be damaged more than the nonmyelinated axons. Electrical shock may induce the greatest trans-membrane potential among the largest diameter nerve fibres and those with the thickest membrane. The injury to the peripheral nerve could result in de-myelination and re-myelination, axonal degeneration and regeneration, loss of nerve fibers (focal, multifocal, diffuse), and endoneural edema (Chizuka et al., 1983).

Yunxia et al. (2003) reported that inflammatory cells and their mediators are known to contribute to neuropathic pain following nerve injury. Mast cells play a key role in non-neural models of inflammation and we propose that mast cells and their mediators (in particular histamine) are important in the development of neuropathic pain. The study suggests that mast cell mediators such as histamine released within hours of nerve injury contribute to the recruitment of leukocytes.

The objective of the present study is to describe the possible alterations occur in sciatic nerve of rats exposed to $220 \mathrm{~V}$ by light and transmission electron microscopy. 


\section{MATERIALS and METHODS}

\section{Materials}

Electrical domestic current:

Alternating current $(220 \mathrm{~V})$ using ordinary wire connected to electrical stabilizer was applied at the thigh region of all investigated rats.

\begin{abstract}
Animals
Twelve rats were purchased from Assiut Lab. Animal House and acclimated for two weeks. These rats were divided in two groups exposed and nonexposed. The exposed rats were subjected to AC current 220 volt for 30 seconds through a wire fixed in the thigh region (gastrocenmous muscles). Rats were dissected after 0,30 , and 60 minutes postexposure.
\end{abstract}

\section{Sampling}

The sciatic nerve was taken immediately after exposure, minced into small pieces and fixed in $4 \%$ cold glutraldehyde. Samples were processed and sectioned used ultratome, dehydrated, and stained with toulidine blue and examined by light microscopy. TEM samples were processed, sectioned and stained with lead citrate and uranyl acetate and examined by Joel X100 CXII TEM at Assiut Electron Microscope Unit and photo-graphed using Digital Camera CCD Jacan, Model XR. Measurements of nerve sheath from both exposed and non-exposed rats were made using the CCD camera and expressed as the Mean \pm SEM.

\section{RESULTS}

Semithin sections stained with toulidine blue showed the normal appearance of sciatic nerve bundles surrounded by perineurium and epineurium. They have axons surrounded with myelin sheath (Figs. 1;2). By thirty minutes of exposure to $220 \mathrm{~V}$, irregularity of the nerve bundles and annulations of the myelin sheath were observed (Fig.3). Moreover, inflammatory cellular reaction was observed in the fascia surrounded the damaged nerve. Mast cells as well as other mononuclear cells were observed in the fascia surrounded the nerve bundles (Fig.4). More irregularity in the shape of the nerve fibers were observed (Fig.5). Transmission electron microscopy of the non-exposed rat sciatic nerve showed axons surrounded by myelin sheath and showann cells. The mean average thickness of the myelin sheath was $(1.41 \pm 0.7) \mu \mathrm{m}$ (Figs 6 ; 7). On the contrary, exposed sciatic nerve by 30 minutes of exposure had marked increase in the thickness of the myelin sheath $(2.32 \pm 0.8) \mu \mathrm{m}$, marked irregularity, fragmentation, and appeared as "bulby" and vacuolated indicating wallerian degeneration Moreover, mast cells with several empty vacuoles were observed (Figs.8;9). By 60 minutes of exposure more fragmentation was observed (Figs. 10; 11).

\section{FIGURE LEGENDS}

Fig.1: Semithin section of control rat sciatic nerve showed bundles of nerve fibers surrounded with perineurum. Toulidine blue. X5.

Fig.2: Higher magnification of Fig.1. Toulidine blue. X10

Fig.3: Section nerve drom rats exposed to $220 \mathrm{~V}$ and collected at 30 minutes showed irregularity of the nerve fibrs (small arrows). Toulidine blue. X10.

Fig.4: Semithin of nerve drom rats exposed to $220 \mathrm{~V}$ and collected at 30 minutes showed accumulation of inflammatory cells mainly mast cell. (Head arrow). Toulidine blue. X40.

Fig.5: Semithin nerve drom rats exposed to $220 \mathrm{~V}$ and collected at 60 minutes showed elongation and irregularity in the shape of the nerve fibas (arrows). Toulidine blue. X40.

Fig.6: TEM of from non exposed rats sciatic nerve showed the normal appearance of axons (Ax), myelin sheath (M), and Shwann cell (Sch). Uranyl acetate and Lead nitrate.

Fig.7: TEM of sciatic nerve drom non exposed rots showed the thickness of the myelin sheath as $(1.41 \pm 0.7) \mu \mathrm{m}$. Uranyl acetate and Lead nitrate.

Fig.8: TEM of sciatic nerve fromexposed rats by 30 minutes showed vacualation or bulby appearance of the myelin sheath (Arrow), and elongated mast cells with empty vacuoles (star). Uranyl acetate and Lead nitrate.

Fig.9: TEM of sciatic nerve from exposed rats by 30 minutes showed the thickness of the myelin sheath as $(2.32 \pm 0.8) \mu \mathrm{m}$. Uranyl acetate and Lead nitrate.

Fig.10: TEM of sciatic nerve from exposed rats by 60 minutes showed marked fragmentation of the myelin sheath. Uranyl acetate and Lead nitrate.

Fig.11: TEM of exposed rat sciatic nerve by 60 minutes showed the thickness of the myelin sheath as $(2.78 \pm 0.8) \mu \mathrm{m}$. Uranyl acetate and Lead nitrate. 


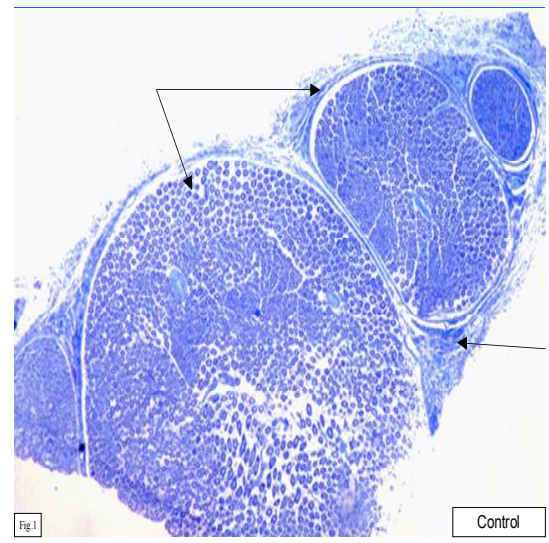

Fig.1

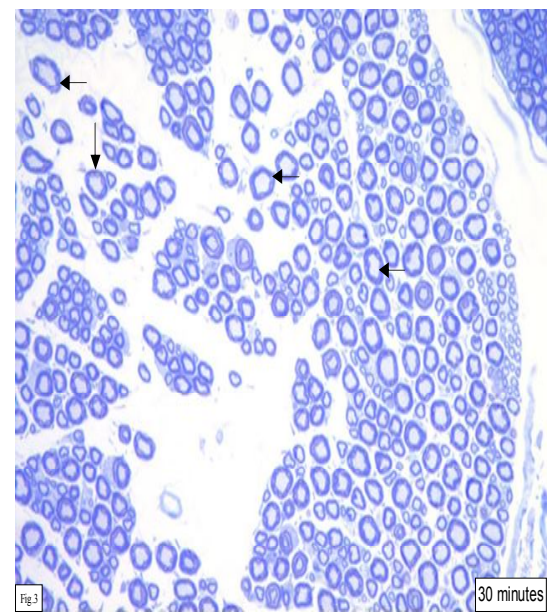

Fig.3

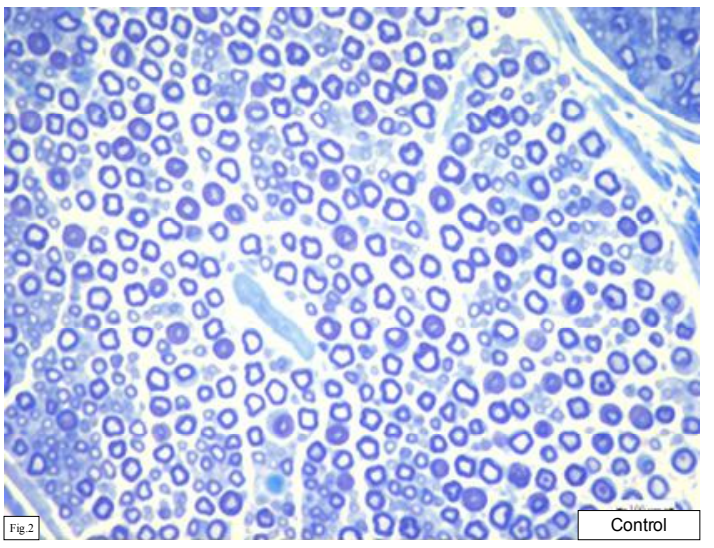

Fig.2

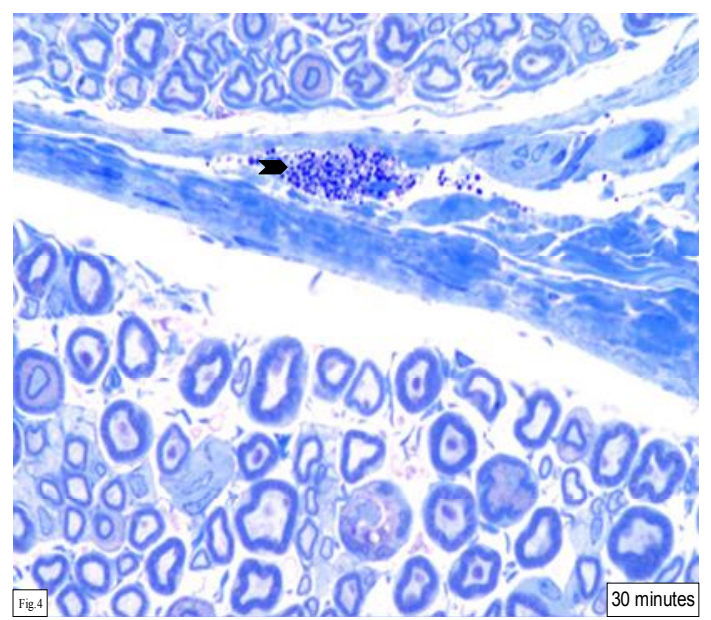

Fig.4

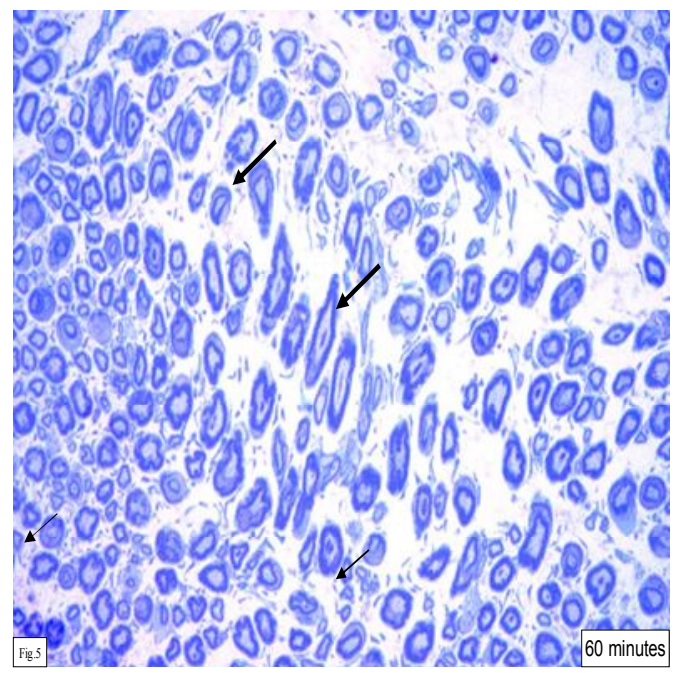

Fig.5 


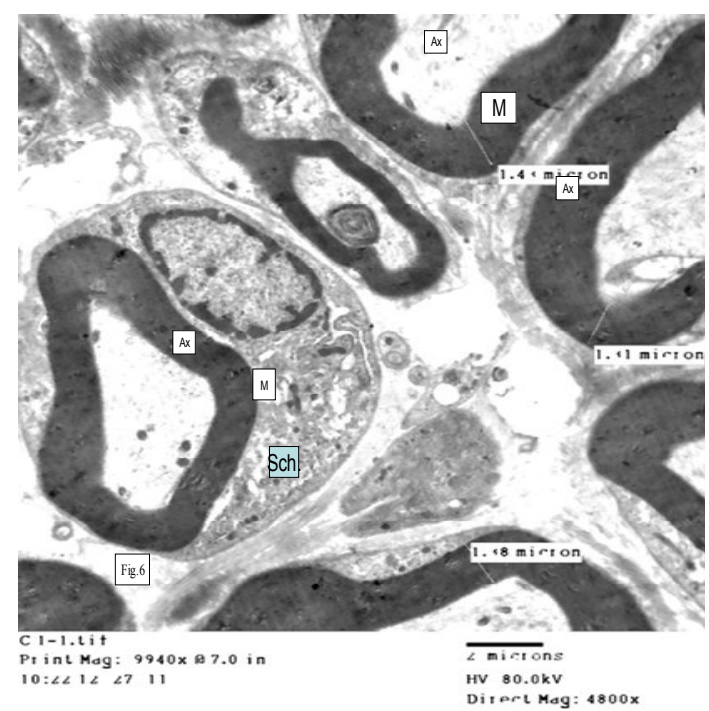

Fig.6

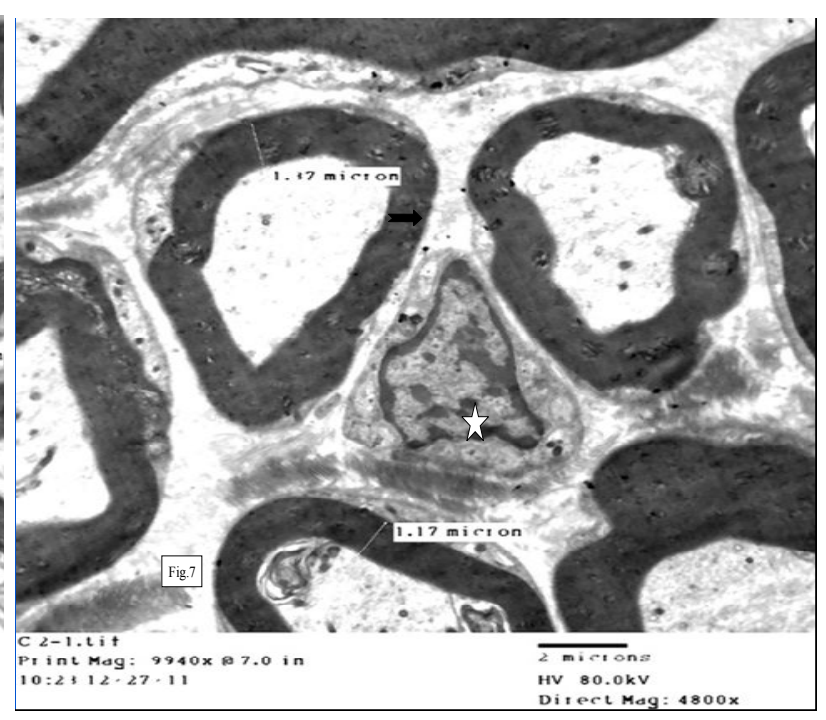

Fig. 7

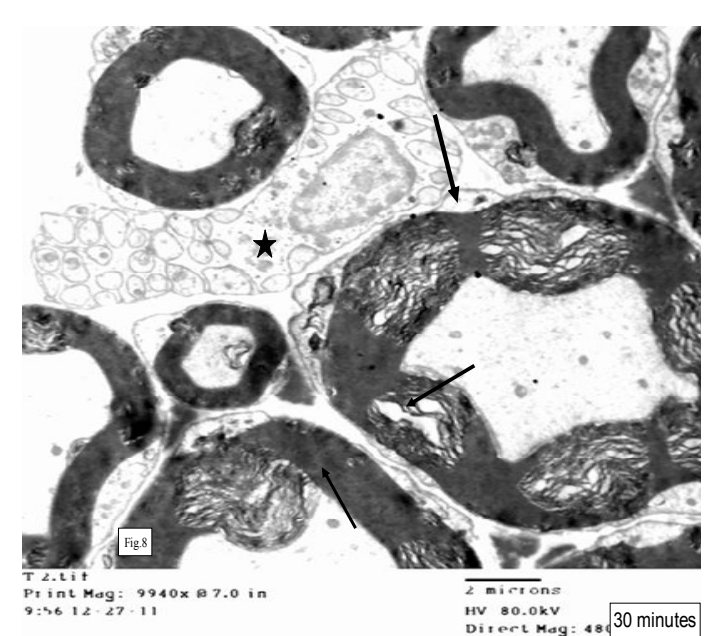

Fig.8

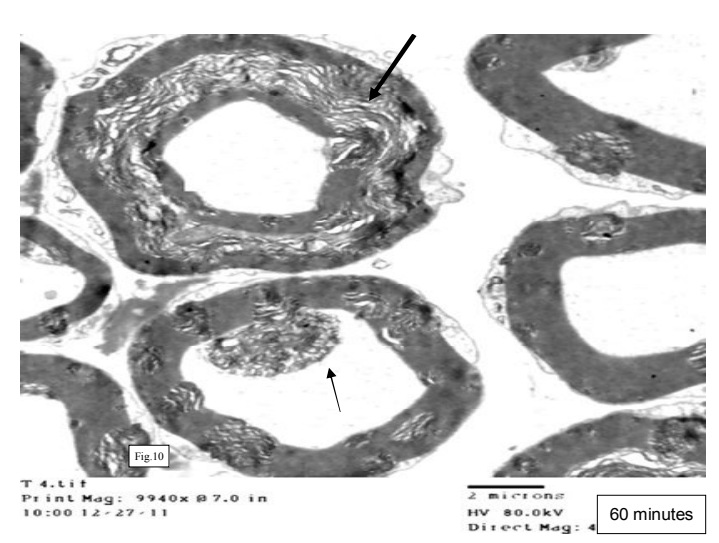

Fig.10

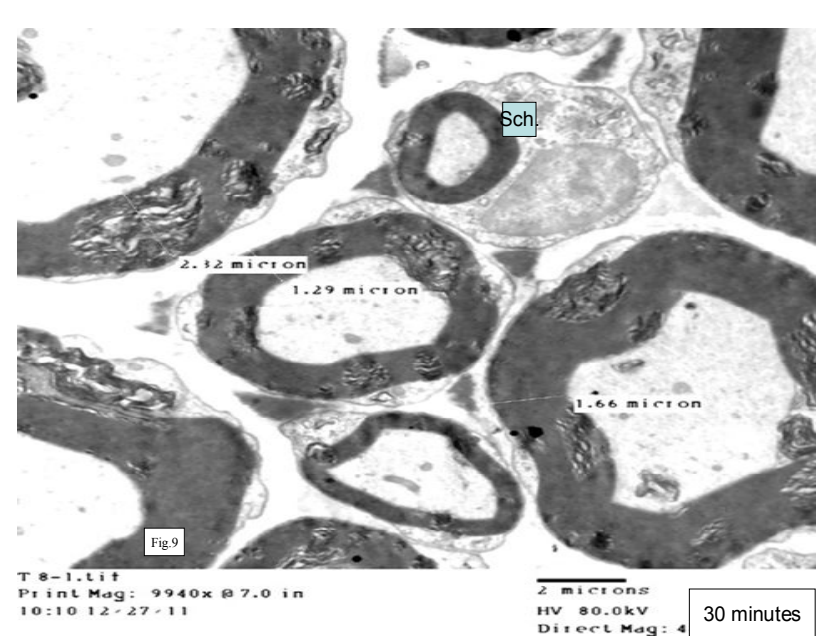

Fig.9

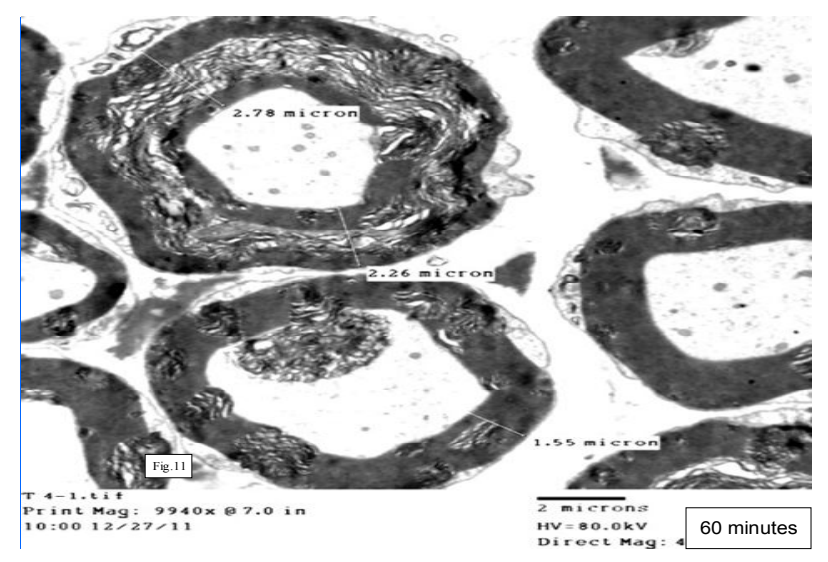

Fig.11 


\section{DISCUSSION}

Kun-Wu et al. (2005) described the histolopathological changes in peripheral nerves exposed to electricity in rats. The blood vessel had embolism within the injured nerve. A large number of nerve fibers experienced Waller degeneration while the myelin sheath was vacuolated. Some nerve fibers experienced Waller degeneration and disintegration. Regenerative myelin appeared in some rats at about the fourth week after injury. The point of entry of the electric currents showed obvious Waller degeneration and disintegration of the myelin sheath, while some nerves showed a regenerated myelin sheath by the second week after injury. The morphology (such as quantity and diameter) of the injured myelin was basically normal by the fourth week after injury. In the present study, vacuolation, disintegration of the nerve fibers were observed at 30 and 60 minutes post-exposure compared to the non-exposed. Moreover, the severity of alteration observed in the nerve fibers was time-dependant.

Mast cells were also observed outside the damaged nerve fibers at 30 minutes post-exposure. Several reports indicated the recruitments of inflammatory cells in the injured peripheral nerves in response to trauma and legation. Degranulation of mast cells was also evident by TEM in the present study. Toews et al. (1998) stated that following injury to the peripheral nervous system, circulating monocytes/macrophages are recruited to the damaged tissue, where they play vital roles during both nerve degeneration and subsequent regeneration. Deborah and helme (1985) mentioned that substance $\mathrm{P}$ is a putative mediator of neurogenic inflammation, where it is postulated to be released from nerve terminals in the skin in response to noxious and electrical stimulation.

In conclusion, this study showed alterations of the sciatic nerve are time dependant and mast cell degranulation was profound and could contributed to the neuropathic pain.

\section{REFERENCES}

Anders, S.; Matschke, J. and Tosokos, M. (2001): Internal current mark in a case of suicide by electrocution, Am. J. Forensic Med., 22 (4), 370-373.

Chizuka Ide; Koujiro Tohyama; Reiko Yokota; Tohru Nitatori and Satoru Onodera (1983). Schwann cell basal lamina and nerve regeneration. Brain Research 288,61-75.

Deborah M. White and Robert D. Helme (1985): Release of substance P from peripheral nerve terminals following electrical stimulation of the sciatic nerve.Brain research 336,27-31.

Kun-Wu Fan; Zhi-Xiang Zhu and Zhi-Yun Den (2005): An experimental model of an electrical injury to the peripheral nerve.Burns.31,731-736.

Toews, Arrel D; Cheri Barrett and Pierre Morell (1998): Monocyte chemoattractant protein 1 is responsible for macrophage recruitment following injury to sciatic nerve. J. of Neural Science Research 53(2), 260-267.

Yunxia Zuo; Nicholas M Perkins; David J Tracey and Carolyn L Geczy (2003): Inflammation and hyperalgesia induced by nerve injury in the rat: a key role of mast cells. Pain 105, 467-479.

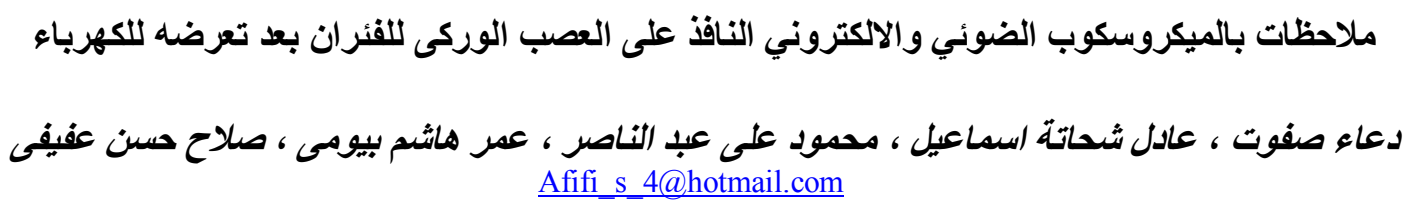

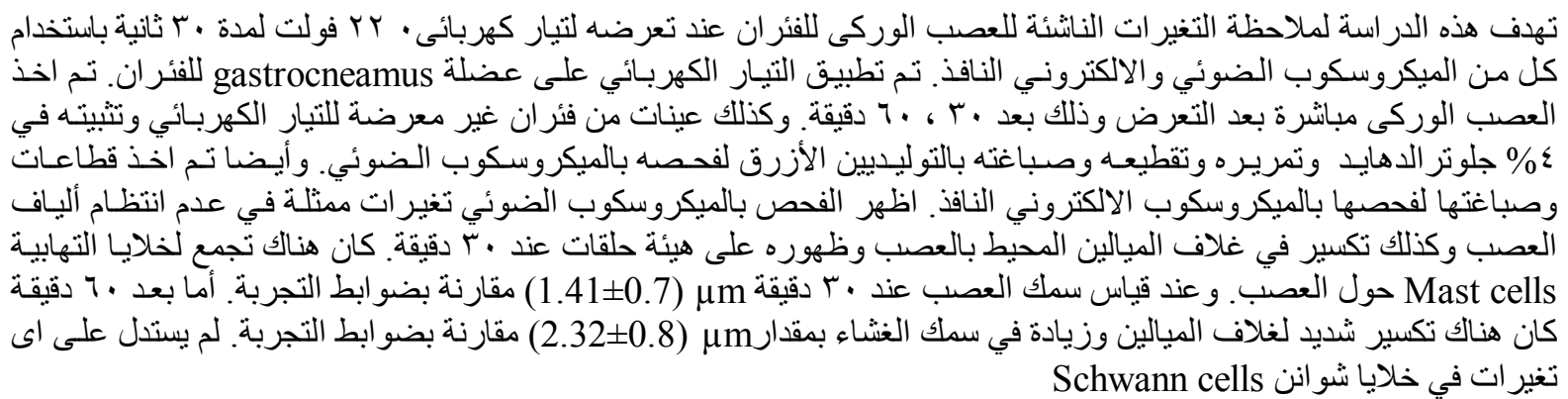

